\title{
Interleaving Schemes for Multidimensional Cluster Errors
}

\author{
Mario Blaum, Senior Member, IEEE, Jehoshua Bruck, Senior Member, IEEE, \\ and Alexander Vardy, Senior Member, IEEE
}

\begin{abstract}
We present two-dimensional and three-dimensional interleaving techniques for correcting two- and three-dimensional bursts (or clusters) of errors, where a cluster of errors is characterized by its area or volume. Correction of multidimensional error clusters is required in holographic storage, an emerging application of considerable importance. Our main contribution is the construction of efficient two-dimensional and three-dimensional interleaving schemes. The proposed schemes are based on $t$ interleaved arrays of integers, defined by the property that every connected component of area or volume $t$ consists of distinct integers. In the two-dimensional case, our constructions are optimal: they have the lowest possible interleaving degree. That is, the resulting $t$-interleaved arrays contain the smallest possible number of distinct integers, hence minimizing the number of codewords required in an interleaving scheme. In general, we observe that the interleaving problem can be interpreted as a graph-coloring problem, and introduce the useful special class of lattice interleavers. We employ a result of Minkowski, dating back to 1904 , to establish both upper and lower bounds on the interleaving degree of lattice interleavers in three dimensions. For the case $t \equiv 0 \bmod 6$, the upper and lower bounds coincide, and the Minkowski lattice directly yields an optimal lattice interleaver. For $t \not \equiv 0 \bmod 6$, we construct efficient lattice interleavers using approximations of the Minkowski lattice.
\end{abstract}

Index Terms-Bursts, chromatic number, clusters, error-correcting codes, lattices, $L_{1}$-distance, multidimensional interleaving, power graphs of $\mathbb{Z}^{3}$.

\section{INTRODUCTION}

A one-dimensional error burst of length $t$ is a set of errors confined to $t$ consecutive locations [28]. In this paper, we generalize the concept of one-dimensional error bursts to two and three dimensions. Namely, we consider errors confined to a connected area or volume of size $t$. Most two-dimensional burst error-correcting codes that have been studied in the literature so far [1], [4], [5], [11], [14], [19], [20], correct

Manuscript received August 30, 1995; revised July 24, 1997. The work of J. Bruck was supported by the NSF Young Investigator Award CCR-9457811, by the Sloan Research Fellowship, and by DARPA and BMDO through an agreement with NASA/OSAT. The work of A. Vardy was supported by the David and Lucile Packard Foundation Fellowship, by the National Science Foundation CAREER Award NCR-9501345, and by the JSEP under Grant N00014-9610129. The material in this paper was presented in part at the IEEE International Symposium on Information Theory, Ulm, Germany, June 1997.

M. Blaum is with the IBM Research Division, Almaden Research Center, San Jose, CA 95120 USA.

J. Bruck is with the California Institute of Technology, Pasadena, CA 91125 USA.

A. Vardy is with the Coordinated Science Laboratory, University of Illinois at Urbana-Champaign, Urbana, IL 61801 USA.

Publisher Item Identifier S 0018-9448(98)00984-5. error bursts of a given rectangular shape, say $t_{1} \times t_{2}$. Several papers study other shapes as well. For instance, a "circular" type of bursts is studied in [2]. In [10], [13], [30], the authors consider metrics given by the rank of an array: a particular case is the correction of "criss-cross" errors. Metrics for different channels, including two-dimensional clusters, are presented in [12].

An important recent application where correction of twodimensional and three-dimensional error clusters is required is holographic storage [21]. Since our results are very much relevant in this context, we briefly review the holographic recording principles in what follows. For further information, we refer the reader to [3], [18], and [31].

Holographic storage: In holographic recording, a laser illuminates a programmable spatial light modulator (SLM), thereby generating an object beam, which represents a twodimensional page of data. The data page consists of a pattern of zeros and ones, where a one is represented by light and a zero by the absence of light. A reference beam, which is a simple plane wave generated by the same laser, interferes with the object beam at a spot on the recording medium and writes a grating pattern, that is the hologram. The original page of data is retrieved at a later time by illuminating the medium with the same reference beam that was used to record it. The data is then collected on a photosensitive array of detectors, known as a charge-coupled device (CCD). Several holograms can be recorded in the same physical spot using a scheme known as angular multiplexing, based on varying the angle of propagation of the reference beam. Such a collection of holograms is called a stack. Since an entire page of data can be retrieved all at once, holographic data storage holds the promise of very high data rate. Furthermore, since many holograms can be recorded in one stack, it also holds the promise of very high data density.

A holographic storage system may be viewed as a noisy channel, which we call the holographic data channel. We define noise as anything that distorts the data signal, whether in a systematic or in a statistical fashion. There are many possible noise sources that affect the performance of this channel. The most dominant source is the loss in diffraction efficiency due to the multiplexing of several holograms in a single stack. It is fairly well accepted [6], [22], [25] that as the number $M$ of holograms written in a stack increases, the diffraction efficiency for retrieving a single hologram decreases as $1 / M^{2}$. The noise due to scatter of the reference beam in the retrieval process is independent of the number of 
holograms, and so the signal-to-noise ratio (SNR) is degraded by the same factor of $1 / M^{2}$. Another noise source is crosstalk from adjacent holograms, but the SNR degradation due to crosstalk is believed to be on the order of $1 / M$, and so appears to be a less serious problem [7], [17]. However, improvements in optics as well as materials may change this situation.

There are many other noise sources. For instance, there may be nonuniformities in the object beam or reference beam. Defects in the storage medium itself can introduce distortions. Since the object beam is really a spatial Fourier transform of the data pattern, the finite extent of the storage medium causes a low-pass filtering effect, so that data content at high spatial frequencies may be lost. Finally, at the detector itself, there are several sources of noise. Quantum noise can cause photon-counting errors. Noise in the camera electronics, as well as rotations and distortions, can cause pixel registration errors, namely, erroneous matching of the CCD pixels to the SLM pixels.

How can we protect the data from these various noise sources? For some problems, such as rotations causing pixel registration errors, fiduciary marks can be imposed to help match CCD pixels to SLM pixels. For other sources of noise, the data can be coded so as to reduce the undesirable effects of the noise.

User data is usually encoded via an error-correction code (ECC) and then a modulation code. The purpose of the modulation code is to adapt the recorded data to the characteristics of the channel-in other words, to "shape" the data in a way that is less likely to be corrupted by noise. For instance, a modulation code can be used to compensate for loss of highfrequency data content [3], [32]. The purpose of the ECC is to correct errors: the ECC adds redundancy to the data, so that error-corrupted data patterns can be retrieved correctly. It is shown in [26] how ECC can be used to fight the phenomenon of $1 / M^{2}$ degradation in SNR, and therefore improve the overall capacity.

Both kinds of coding - modulation and error-correctionare performed digitally. On the other hand, the outputs from the CCD are essentially analog (photon counts). So, before the modulation decoder can process the data, a detection scheme must make a digital decision on the outputs from the CCD. Ultimately, the detection scheme, the modulation code, and the ECC should be designed in concert so that they work well together. For instance, the detection scheme can use information based on the constraints of the modulation code, and the ECC may need to correct clusters of errors caused by the modulation decoder.

Multidimensional interleaving: A conventional magnetic or optical storage system is usually regarded as a onedimensional channel since each track carries the information contained in a one-dimensional stream of data. If one takes into account the effects of neighboring tracks, then such a system may be also regarded as a two-dimensional channel. Since holograms represent inherently two-dimensional pages of data, the holographic data channel is naturally viewed as a two-dimensional channel. With angular multiplexing taken into account, it becomes a three-dimensional channel. In general, it is fair to say that the theory and practice of coding and detection is much better developed for one-dimensional channels than for higher dimensional channels.

The most common approach to dealing with one-dimensional error bursts is interleaving. The idea is to assign consecutive symbols in a data sequence to a number of separate codewords (or codes). For example, to implement the correction of bursts of length 3 , one can use three different codewords in a single-error-correcting code, while encoding (or interleaving) the one-dimensional data sequence as follows: $123123123123123123123123123123 \cdots$. Here, the symbols 1,2 , and 3 correspond to the first, second, and third codewords, respectively. This straightforward interleaving scheme, requiring $t$ different single-error-correcting codes to correct bursts of length $t$, is optimal in the sense that there is no other interleaving scheme that can correct a burst of length up to $t$ and requires less than $t$ different codes.

However, in higher dimensions, it is not at all obvious how to interleave a minimal number of codes so that any cluster of size $t$ can be corrected. Our main contribution is the construction of efficient two-dimensional and threedimensional interleaving schemes. In the two-dimensional case, our constructions are optimal in the sense that they require the smallest possible number of distinct codes. We note here that a related construction, with the constraint that the error burst has a rectangular shape, was presented in [8].

Next, we formalize the problem of constructing twodimensional interleaving schemes by introducing appropriate notation. This notation is further generalized to three dimensions in Section III. We will refer to locations, or positions, in a finite multidimensional array as elements of the array. We say that an element $(x, y)$ in a two-dimensional array is connected to elements $(x+1, y),(x-1, y),(x, y+1)$, and $(x, y-1)$, provided those elements exist. A path of length $n$ from an element $\mathcal{E}_{0}$ to and element $\mathcal{E}_{n}$ in a twodimensional array is a sequence of $n+1$ distinct elements $\mathcal{E}_{0}, \mathcal{E}_{1}, \cdots, \mathcal{E}_{n}$, such that $\mathcal{E}_{i}$ is connected to $\mathcal{E}_{i-1}$ for all $i=1,2, \cdots, n$.

Definition 1.1: A set $\mathcal{S}$ of $t$ elements in a two-dimensional array is a cluster of size $t$, if any two elements in $\mathcal{S}$ belong to a path that is contained in $\mathcal{S}$.

The concept of a cluster of size $t$ generalizes to two dimensions the concept of a burst of size $t$ in one dimension. For example, the $*$ 's in the array below constitute a cluster of size 7 .

\begin{tabular}{|c|c|c|c|c|c|c|}
\hline 0 & 0 & 0 & 0 & 0 & 0 & 0 \\
\hline 0 & $*$ & $*$ & $*$ & 0 & 0 & 0 \\
\hline 0 & 0 & $*$ & $*$ & $*$ & 0 & 0 \\
\hline 0 & 0 & 0 & $*$ & 0 & 0 & 0 \\
\hline
\end{tabular}

As we shall see in Section III, the foregoing definition of a cluster easily extends to multiple dimensions. We now define $t$-interleaved arrays and interleaving degree.

Definition 1.2: Let $A$ be a two-dimensional array of integers; that is, the elements of $A$ are labeled by integers. We say 
that $A$ is $t$-interleaved if every cluster of size $t$ in $A$ is labeled by $t$ distinct integers. The total number of distinct integers used to label the elements of $A$ is called the interleaving degree of $A$ and denoted by $\operatorname{deg} A$.

Notice that if the integers in a $t$-interleaved array represent different codewords, as in the one-dimensional case, then any error cluster of size up to $t$ can be corrected. Furthermore, if the interleaving degree is $m$, and each of the $m$ codewords is drawn from a code that corrects at least $\tau$ errors, then any $\tau$ clusters of size up to $t$ can be corrected.

Example 1.1: The following array is 3 -interleaved with interleaving degree 5 .

\begin{tabular}{|l|l|l|l|l|l|l|}
\hline 0 & 1 & 2 & 3 & 4 & 0 & 1 \\
\hline 3 & 4 & 0 & 1 & 2 & 3 & 4 \\
\hline 1 & 2 & 3 & 4 & 0 & 1 & 2 \\
\hline 4 & 0 & 1 & 2 & 3 & 4 & 0 \\
\hline 2 & 3 & 4 & 0 & 1 & 2 & 3 \\
\hline
\end{tabular}

In terms of the foregoing notation, our goal in this paper may be concisely described as constructing $t$-interleaved arrays, in two and three dimensions, with minimal interleaving degree. We point out that this goal can also be phrased as a graphcoloring problem, where the underlying graph is the grid graph $G$ on the set of vertices $V=\mathbb{Z}^{2}$, or $V=\mathbb{Z}^{n}$ for higher dimensions. Different integers in the array can be interpreted as different colors, and we want to make sure that the coloring is such that two vertices of the same color never appears twice in a cluster of size $t$. This is equivalent to coloring the power graph $G^{t}$ in such a way that adjacent vertices have different colors. Notice that $G^{t}$ is a power of a Cartesian product of graphs. Determining the chromatic number of a Cartesian product is easy: it is well-known [33], [34] that

$$
\chi\left(G_{1} \otimes G_{2}\right)=\max \left\{\chi\left(G_{1}\right), \chi\left(G_{2}\right)\right\}
$$

and hence $\chi(G)=2$ for all $n$. However, determining the chromatic number of a power is not at all easy. To the best of our knowledge, the chromatic numbers of the kind of graphs that we are interested in have not been previously studied in the literature.

Notice that in the one-dimensional case, the minimal interleaving degree $t$ coincides with the size of the burst we want to correct. This is no longer true in two or more dimensions, as we will see in what follows. In the next section we present optimal two-dimensional interleaving schemes. In Section III we generalize our methods to three (and more) dimensions. In Section IV, we introduce lattice interleavers, and analyze their properties.

\section{TWO-DIMENSIONAL INTERLEAVING}

In this section, we present two optimal constructions for $t$ interleaved arrays in two dimensions. We start by presenting lower bounds on the interleaving degree of $t$-interleaved arrays.

\section{A. Lower Bounds}

The following theorem is a simple sphere-packing lower bound on the interleaving degree. As we shall see later in this section, this bound is exact in two dimensions.

Theorem 2.1: Let $A$ be a sufficiently large two-dimensional $t$-interleaved array. Then the interleaving degree of $A$ is bounded by

$$
\operatorname{deg} A \geq \begin{cases}\frac{t^{2}}{2}, & \text { for } t \text { even } \\ \frac{t^{2}+1}{2}, & \text { for } t \text { odd }\end{cases}
$$

Proof: For every $t \geq 1$, we define the appropriate twodimensional sphere $\mathcal{S}_{2, t}$. The key idea of the proof is to show that any two elements in such a sphere belong to some cluster of size at most $t$. Hence all the elements in a sphere $\mathcal{S}_{2, t}$ must be assigned distinct integers in a $t$-interleaved array. The spheres $\mathcal{S}_{2, t}$ are defined recursively, while distinguishing between odd and even $t$. Consider an array. The sphere $\mathcal{S}_{2,1}$ is a single element in the array. The sphere $\mathcal{S}_{2,2}$ is a $1 \times 2$ subarray. The sphere $\mathcal{S}_{2, t+2}$ is constructed from $\mathcal{S}_{2, t}$ by appending to $\mathcal{S}_{2, t}$ all the elements of the array that are connected to some element of $\mathcal{S}_{2, t}$.

Next we show that, for every $t$, any two elements of $\mathcal{S}_{2, t}$ are connected by a path of length at most $t-1$ that is wholly contained in $\mathcal{S}_{2, t}$. The proof of this claim is by induction on $t$. The claim is obviously true for $t=1$ and $t=2$. Now assume that it is true for $t$, and consider the sphere $\mathcal{S}_{2, t+2}$. Let $\alpha, \beta$ be two arbitrary elements of $\mathcal{S}_{2, t+2}$. By construction, $\mathcal{S}_{2, t}$ is contained in $\mathcal{S}_{2, t+2}$. If $\alpha$ and $\beta$ are both elements of $\mathcal{S}_{2, t}$, then by induction hypothesis there is a path of length at most $t-1$ between them. Otherwise, we observe that by construction, an element of $\mathcal{S}_{2, t+2}$ that is not also in $\mathcal{S}_{2, t}$ is connected to an element of $\mathcal{S}_{2, t}$. Hence, there is a path of length at most $t+1$ between $\alpha$ and $\beta$, which proves the induction step.

It follows that any $\alpha, \beta \in \mathcal{S}_{2, t}$ belong to some cluster of size at most $t$, namely, the path of length $\leq t-1$ that connects them. Thus all the elements of $\mathcal{S}_{2, t}$ must be labeled distinctly in a $t$ interleaved array, and $\operatorname{deg} A \geq\left|\mathcal{S}_{2, t}\right|$. It is now straightforward to verify that $\left|\mathcal{S}_{2, t}\right|=\frac{1}{2} t^{2}$ for even $t$, and $\left|\mathcal{S}_{2, t}\right|=\frac{1}{2}\left(t^{2}+1\right)$ for odd $t$, which completes the proof of the theorem.

Example 2.1: The first six spheres $\mathcal{S}_{2, t}$ are labeled by in the following:
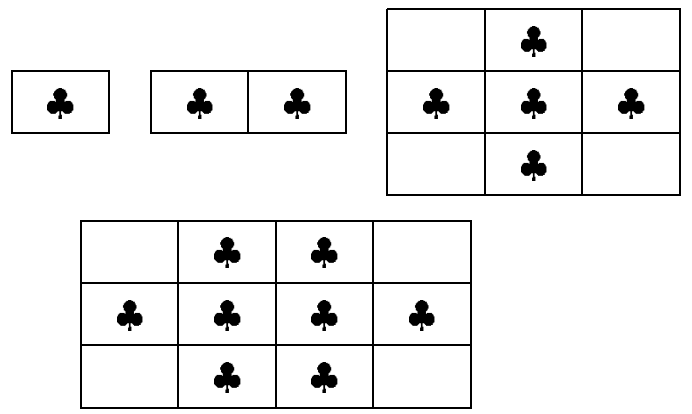


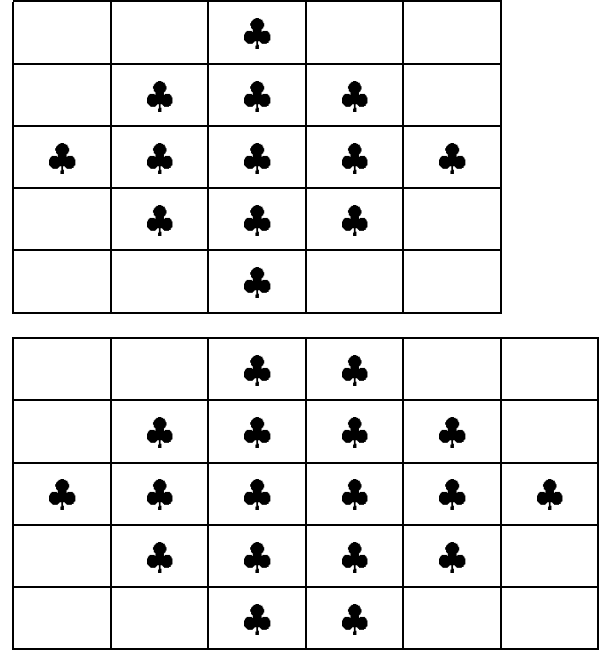

Notice that our proof of Theorem 2.1 also shows that two elements $\left(x_{1}, y_{1}\right)$ and $\left(x_{2}, y_{2}\right)$ in a two-dimensional array belong to the same cluster of size at most $t$, if and only if the $L_{1}$ distance between them is strictly less than $t$, namely, if $\left|x_{1}-x_{2}\right|+\left|y_{1}-y_{2}\right|<t$. This observation further shows that the "spheres" that we have defined as $\mathcal{S}_{2, t}$ are indeed spheres of radius $\frac{1}{2}(t-1)$ in the $L_{1}$ metric, centered at the origin. The distinction between the odd and even cases in this context may be expressed by indexing the arrays with $(x, y) \in \mathbb{Z}^{2}$ for odd $t$, and with $(x, y) \in\left(\frac{1}{2}+\mathbb{Z}\right) \times \mathbb{Z}$ for even $t$. For more details on this, see the next section.

\section{B. Constructions}

Next we present elementary constructions of two-dimensional $t$-interleaved arrays with optimal interleaving degree. Namely, the interleaving degree of both constructions attains the lower bound of Theorem 2.1. First we describe what we call the toroidal interleaving scheme.

Construction 2.1: Let $A$ be a two-dimensional array, and let $m$ be a positive integer. We label the coordinates of the array toroidally on $m$, namely, we assign the same label to elements $(x, y)$ and $\left(x^{\prime}, y^{\prime}\right)$ of $A$ if $x^{\prime} \equiv x$ and $y^{\prime} \equiv y$ modulo $m$. Let $b$ be a fixed positive integer. Then, for each $a=0,1, \cdots, m-1$, the elements $(x, a+x b)$, with both coordinates taken modulo $m$, are labeled by the integer $a$.

Example 2.2: Consider a $4 \times 6$ array; Construction 2.1 with $m=2$ and $b=1$ produces the following labeling of the array:

\begin{tabular}{|l|l|l|l|l|l|}
\hline 0 & 1 & 0 & 1 & 0 & 1 \\
\hline 1 & 0 & 1 & 0 & 1 & 0 \\
\hline 0 & 1 & 0 & 1 & 0 & 1 \\
\hline 1 & 0 & 1 & 0 & 1 & 0 \\
\hline
\end{tabular}

It is easy to verify that the array above is 2-interleaved. Similarly, if we consider a $5 \times 10$ array with $m=5$ and $b=3$, we obtain:

\begin{tabular}{|l|l|l|l|l|l|l|l|l|l|}
\hline 0 & 1 & 2 & 3 & 4 & 0 & 1 & 2 & 3 & 4 \\
\hline 2 & 3 & 4 & 0 & 1 & 2 & 3 & 4 & 0 & 1 \\
\hline 4 & 0 & 1 & 2 & 3 & 4 & 0 & 1 & 2 & 3 \\
\hline 1 & 2 & 3 & 4 & 0 & 1 & 2 & 3 & 4 & 0 \\
\hline 3 & 4 & 0 & 1 & 2 & 3 & 4 & 0 & 1 & 2 \\
\hline
\end{tabular}

The reader can easily verify that the above array is 3interleaved.

It is obvious from the definition of Construction 2.1 that the interleaving degree is precisely $m$. Furthermore, as we can see in Example 2.2, given an array $A$ labeled by Construction 2.1, in order to find if the array is $t$-interleaved, it is enough to consider any $m \times m$ subarray. This is so because the labeling of the whole array $A$ can then be obtained by tiling it with copies of the $m \times m$ subarray. Whether the $m \times m$ subarray, and hence the entire array $A$, is $t$-interleaved depends only on the choice of the integer $b$. Thus the problem reduces to an appropriate choice for $b$. These observations lead to the following two theorems.

Theorem 2.2: Let $t$ be an odd integer. Then Construction 2.1 with parameters $b=t$ and $m=\frac{1}{2}\left(t^{2}+1\right)$ produces a $t$-interleaved array.

Theorem 2.3: Let $t$ be an even integer. Then Construction 2.1 with parameters $b=t+1$ and $m=\frac{1}{2} t^{2}$ produces a $t$-interleaved array.

We defer the proof of Theorem 2.2 and Theorem 2.3 to Section IV. Both theorems can be proved in elementary terms; however, a more concise proof follows directly from the general results on lattice interleavers developed in Section IV.

Example 2.3. Consider the case $t=4$. According to Theorem 2.3, we should take $m=8$ and $b=5$ in Construction 2.1. Therefore, to ensure that an arbitrary array $A$ is 4-interleaved, we can tile it with copies of the following $8 \times 8$ array:

\begin{tabular}{|l|l|l|l|l|l|l|l|}
\hline 0 & 1 & 2 & 3 & 4 & 5 & 6 & 7 \\
\hline 3 & 4 & 5 & 6 & 7 & 0 & 1 & 2 \\
\hline 6 & 7 & 0 & 1 & 2 & 3 & 4 & 5 \\
\hline 1 & 2 & 3 & 4 & 5 & 6 & 7 & 0 \\
\hline 4 & 5 & 6 & 7 & 0 & 1 & 2 & 3 \\
\hline 7 & 0 & 1 & 2 & 3 & 4 & 5 & 6 \\
\hline 2 & 3 & 4 & 5 & 6 & 7 & 0 & 1 \\
\hline 5 & 6 & 7 & 0 & 1 & 2 & 3 & 4 \\
\hline
\end{tabular}

For $t=5$, we should take $m=13$ and $b=5$ according to Theorem 2.2. Therefore, to ensure that an array $A$ is 5interleaved, we can tile it with the $13 \times 13$ array, shown at the top of the following page: 


\begin{tabular}{|c|c|c|c|c|c|c|c|c|c|c|c|c|}
\hline 0 & 1 & 2 & 3 & 4 & 5 & 6 & 7 & 8 & 9 & 10 & 11 & 12 \\
\hline 8 & 9 & 10 & 11 & 12 & 0 & 1 & 2 & 3 & 4 & 5 & 6 & 7 \\
\hline 3 & 4 & 5 & 6 & 7 & 8 & 9 & 10 & 11 & 12 & 0 & 1 & 2 \\
\hline 11 & 12 & 0 & 1 & 2 & 3 & 4 & 5 & 6 & 7 & 8 & 9 & 10 \\
\hline 6 & 7 & 8 & 9 & 10 & 11 & 12 & 0 & 1 & 2 & 3 & 4 & 5 \\
\hline 1 & 2 & 3 & 4 & 5 & 6 & 7 & 8 & 9 & 10 & 11 & 12 & 0 \\
\hline 9 & 10 & 11 & 12 & 0 & 1 & 2 & 3 & 4 & 5 & 6 & 7 & 8 \\
\hline 4 & 5 & 6 & 7 & 8 & 9 & 10 & 11 & 12 & 0 & 1 & 2 & 3 \\
\hline 12 & 0 & 1 & 2 & 3 & 4 & 5 & 6 & 7 & 8 & 9 & 10 & 11 \\
\hline 7 & 8 & 9 & 10 & 11 & 12 & 0 & 1 & 2 & 3 & 4 & 5 & 6 \\
\hline 2 & 3 & 4 & 5 & 6 & 7 & 8 & 9 & 10 & 11 & 12 & 0 & 1 \\
\hline 10 & 11 & 12 & 0 & 1 & 2 & 3 & 4 & 5 & 6 & 7 & 8 & 9 \\
\hline 5 & 6 & 7 & 8 & 9 & 10 & 11 & 12 & 0 & 1 & 2 & 3 & 4 \\
\hline
\end{tabular}

Theorems 2.2 and 2.3 give optimal two-dimensional interleaving schemes for any $t$, since they meet the sphere-packing lower bound of Theorem 2.1. We now present an alternative optimal construction for the case of even $t$. This construction is again elementary.

Construction 2.2: Let $t=2 k$ be even. We take two $k \times k$ arrays $\mathcal{C}_{1}$ and $\mathcal{C}_{2}$ and label every element in $\mathcal{C}_{1}, \mathcal{C}_{2}$ distinctly, using some $\frac{1}{2} t^{2}=2 k^{2}$ distinct integers, say $0,1, \cdots, 2 k^{2}-1$. The $t$-interleaved array $A$ then consists of the chess-board-like tiling using the arrays $\mathcal{C}_{1}$ and $\mathcal{C}_{2}$.

Example 2.4. For $t=6$, Construction 2.2 produces the following:

$\mathcal{C}_{1}=$\begin{tabular}{|l|l|l|}
\hline 0 & 1 & 2 \\
\hline 3 & 4 & 5 \\
\hline 6 & 7 & 8 \\
\hline
\end{tabular}

$\mathcal{C}_{2}=$\begin{tabular}{|c|c|c|}
\hline 9 & 10 & 11 \\
\hline 12 & 13 & 14 \\
\hline 15 & 16 & 17 \\
\hline
\end{tabular}

and
Theorem 2.4: For every even $t$, the array $A$ in Construction 2.2 is $t$-interleaved.

Proof: A path connecting any two elements with the same label, say the label 0 in array $\mathcal{C}_{1}$, must pass through the other array $\mathcal{C}_{2}$. Hence, the length of such a path is at least $t$.

\section{Three-DimensionAl INTERLEAVING}

In this section we extend the results of the previous section to the case of three dimensions. These results can be further extended to higher dimensions, but we will not do it here.

First, we briefly adapt some of the definitions given in Section I to the three-dimensional case. We say that an element $(x, y, z)$ in a three-dimensional array is connected to elements $(x+1, y, z),(x-1, y, z),(x, y+1, z)$, $(x, y-1, z),(x, y, z+1)$, and $(x, y, z-1)$, provided those elements exist. Notice that this definition trivially extends to higher dimensions. In general, in an $n$-dimensional array, an element is connected to $2 n$ other elements of the array (the kissing number of $\mathbb{Z}^{n}$ is $2 n$ ). With this new meaning of the

$A=$\begin{tabular}{|c|c|c|c|c|c|c|c|c|c|c|c|}
\hline 0 & 1 & 2 & 9 & 10 & 11 & 0 & 1 & 2 & 9 & 10 & 11 \\
\hline 3 & 4 & 5 & 12 & 13 & 14 & 3 & 4 & 5 & 12 & 13 & 14 \\
\hline 6 & 7 & 8 & 15 & 16 & 17 & 6 & 7 & 8 & 15 & 16 & 17 \\
\hline 9 & 10 & 11 & 0 & 1 & 2 & 9 & 10 & 11 & 0 & 1 & 2 \\
\hline 12 & 13 & 14 & 3 & 4 & 5 & 12 & 13 & 14 & 3 & 4 & 5 \\
\hline 15 & 16 & 17 & 6 & 7 & 8 & 15 & 16 & 17 & 6 & 7 & 8 \\
\hline
\end{tabular}


term "connected elements," the definitions of path and cluster remain unchanged. Similarly, Definition 1.2 extends in the obvious way to three-dimensional arrays.

\section{A. Lower Bounds}

As in the previous section, we start with a sphere-packing lower bound. In contrast with the two-dimensional case, however, we will show later in this section that the sphere-packing bound is not exact in three dimensions.

Theorem 3.1: Let $A$ be a sufficiently large three-dimensional $t$-interleaved array. Then the interleaving degree of $A$ is bounded by:

$$
\operatorname{deg} A \geq \begin{cases}\frac{t^{3}+2 t}{6}, & \text { for } t \text { even } \\ \frac{t^{3}+5 t}{6}, & \text { for } t \text { odd }\end{cases}
$$

Proof: The proof is similar to that of Theorem 2.1. We again define the spheres $\mathcal{S}_{3, t}$ recursively, while distinguishing between odd and even $t$. Consider a three-dimensional array. The sphere $\mathcal{S}_{3,1}$ is a single element in the array. The sphere $\mathcal{S}_{3,2}$ is a $1 \times 2$ subarray. The sphere $\mathcal{S}_{3, t+2}$ is constructed from $\mathcal{S}_{3, t}$ by appending to $\mathcal{S}_{3, t}$ all the elements of the array that are connected to some element of $\mathcal{S}_{3, t}$. An inductive argument similar to the one used in Theorem 2.1 shows that $\operatorname{deg} A \geq\left|\mathcal{S}_{3, t}\right|$ in any three-dimensional $t$-interleaved array $A$. Finally, it is straightforward to verify that $\left|\mathcal{S}_{2, t}\right|=\frac{1}{6}\left(t^{3}+2 t\right)$ for even $t$, and $\left|\mathcal{S}_{2, t}\right|=\frac{1}{6}\left(t^{3}+5 t\right)$ for odd $t$, which completes the proof of the theorem.

Example 3.1. The first six spheres $\mathcal{S}_{3, t}$ are depicted below

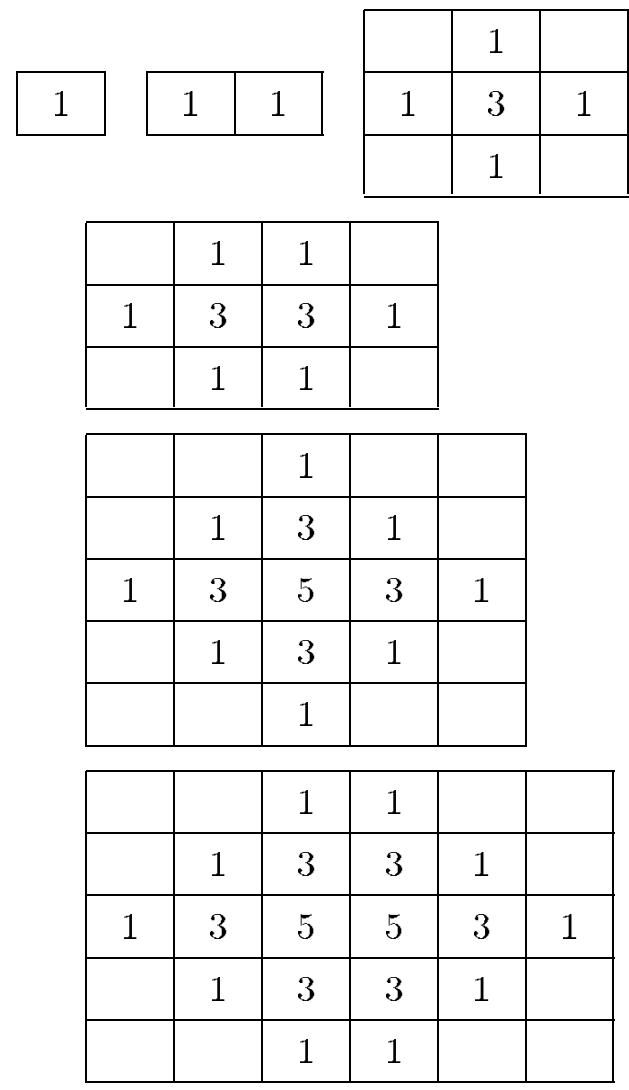

The arrays in (3) represent the projections of three-dimensional spheres on the plane of the paper. These projections are two-dimensional spheres, as described in the previous section. The numbers in (3) indicate how many elements in the three-dimensional sphere project on the corresponding position of the two-dimensional sphere. For example, the number 3 indicates that there is one element above and one element below the plane of the paper.

It might be worthwhile to provide a general expression for the volume of an $n$-dimensional sphere $\mathcal{S}_{n, t}$. For odd $t=2 k+1$, this is given by:

$$
\left|\mathcal{S}_{n, 2 k+1}\right|=\sum_{i=0}^{\min \{n, k\}} 2^{i}\left(\begin{array}{c}
n \\
i
\end{array}\right)\left(\begin{array}{c}
k \\
i
\end{array}\right)
$$

while for even $t=2 k$, we have

$$
\left|\mathcal{S}_{n, 2 k}\right|=\left|\mathcal{S}_{n, 2 k-1}\right|+\left|\mathcal{S}_{n-1,2 k-1}\right| \text {. }
$$

The proof of (4) is straightforward and may be found in [15], while the proof of the above expression for $\left|\mathcal{S}_{n, 2 k}\right|$ amounts to a simple induction on the dimension $n$. Generalizing the arguments of Theorems 2.1 and 3.1, we immediately obtain that the expression in (4), and its extension for even $t$, are lower bounds on the interleaving degree of an $n$-dimensional $t$-interleaved array $A$.

\section{B. Constructions}

We now provide some constructions that give upper bounds on the interleaving degree of a three-dimensional $t$-interleaved array. In most cases, we cannot attain the sphere-packing lower bound of Theorem 3.1. In at least one case, we know that this bound is, in fact, unattainable, as will be shown in the next subsection. In general, it is an open problem to determine the exact bounds for $n=3$.

In what follows, we describe a toroidal interleaving scheme, which is a generalization of the one given in the previous section.

Construction 3.1: Let $A$ be a three-dimensional array, and let $m$ be a positive integer. We label the coordinates of $A$ toroidally on $m$, namely, we assign the same label to elements $(x, y, z)$ and $\left(x^{\prime}, y^{\prime}, z^{\prime}\right)$ of $A$ if $x^{\prime} \equiv x, y^{\prime} \equiv y$, and $z^{\prime} \equiv z$ modulo $m$. Let $b, c$ be fixed integers. Then, for each $a=0,1, \cdots, m-1$, the elements $(x, y, a+x b+y c)$, with all coordinates taken modulo $m$, are labeled by the integer $a$.

Construction 3.1 gives a three-dimensional interleaving scheme with interleaving degree $m$. Let $t$ denote the minimum Lee distance between the elements labeled by the integer $a$, all contained in an $m \times m \times m$ torus. Then it is easy to see that the array labeled by Construction 3.1 is $t$-interleaved. Since elements with different labels $a=0,1, \cdots, m-1$ are translates of each other, it is enough to consider the case $a=0$ to determine the minimum Lee distance in the $m \times m \times m$ torus.

Example 3.2: Consider Construction 3.1, with the following parameters: $m=7, b=2$, and $c=3$. The elements labeled by $a=0$ are $(x, y, 2 x+3 y)$, with all coordinates taken modulo 7. Explicitly, they are as shown at the bottom of this page. 
TABLE I

Bounds on the InTERLEAving Degree of Three-Dimensional $t$-InTERLEAVED ARrays

\begin{tabular}{|c|c|c|c|c|}
\hline 1 & $\begin{array}{l}\text { Sphere-packing } \\
\text { Lower Bound }\end{array}$ & $\begin{array}{r}\text { Upper B } \\
\text { Construction } 31\end{array}$ & $\begin{array}{l}\text { Ind } \\
\qquad(b, c)\end{array}$ & $\begin{array}{l}\text { Upper Bound } \\
\text { Construction } 3.2\end{array}$ \\
\hline 2 & 2 & 2 & $(1,1)$ & \\
\hline 3 & 7 & 7 & $(2,3)$ & \\
\hline 4 & 12 & 12 & $(3,5)$ & 16 \\
\hline$\overline{5}$ & $2 \pi$ & 27 & $(4,10)$ & \\
\hline 6 & 38 & 38 & $(\bar{t}: 11)$ & 56 \\
\hline 7 & 6.3 & 70 & $(16,25)$ & \\
\hline 8 & 88 & 92 & $(9.39)$ & 96 \\
\hline 9 & 129 & 145 & $(9.61)$ & \\
\hline 10 & 170 & 190 & $(9.71)$ & 216 \\
\hline 11 & $2: 31$ & 260 & $(40,94)$ & \\
\hline 12 & 292 & 312 & $(13.115)$ & 304 \\
\hline 13 & 377 & 421 & $(16,182)$ & \\
\hline 14 & 462 & 486 & $(41,57)$ & 260 \\
\hline 15 & 575 & 635 & $(146,274)$ & \\
\hline 16 & 688 & 724 & $(49,79)$ & 736 \\
\hline
\end{tabular}

It can be readily verified that the minimum Lee weight is 3 . Therefore, the resulting array is 3 -interleaved, namely, every cluster of size $\leq 3$ is labeled distinctly. Since for $t=3$, the lower bound on the interleaving degree is 7 , this construction is optimal.

In order to obtain the maximal $t$ from Construction 3.1, we optimize over all possible values of $b$ and $c$. This gives an upper bound on the interleaving degree, for a given $t$. In Table I, we have compiled lower bounds based on Theorem 3.1 and upper bounds based on Construction 3.1, that were obtained by computer search. We also include in Table I the values of $b$ and $c$ that optimize the construction. Of course, these values are not necessarily unique.

We describe next an interleaving scheme for even $t$, that is somewhat similar to Construction 2.2 given in the previous section.

Construction 3.2: The construction is recursive in $t$. Assume that $t$ is even, and let $A_{t / 2}$ be a $(t / 2)$-interleaved array with interleaving degree $m$. We replace each label $a=0,1, \cdots, m-1$ in $A_{t / 2}$ by a $2 \times 2 \times 2$ array $\mathcal{C}_{a}$, with all of $\mathcal{C}_{0}, \mathcal{C}_{1}, \cdots, \mathcal{C}_{m-1}$ labeled distinctly. The result is an array $A_{t}$ with interleaving degree $2^{3} \mathrm{~m}$.

Using an argument similar to the one used in the proof of Theorem 2.4, one can show that the array $A_{t}$ resulting from Construction 3.2 is $t$-interleaved.

Example 3.3. Consider Construction 3.2 with $t=4$. Thus $A_{t / 2}$ is a 2-interleaved array, and we can use an optimal interleaving scheme for $A_{t / 2}$, which has interleaving degree $m=2$. For example, array $A_{t / 2}$ may be tiled by the $2 \times 2 \times 2$ torus:

\begin{tabular}{|l|l|}
\hline 0 & 1 \\
\hline 1 & 0 \\
\hline
\end{tabular}

\begin{tabular}{|l|l|}
\hline 1 & 0 \\
\hline 0 & 1 \\
\hline
\end{tabular}

with the two $2 \times 2$ arrays above representing the two planes of the torus. According to Construction 3.2, we replace each 0

\begin{tabular}{|c|c|c|c|c|c|c|}
\hline$(0,0,0)$ & $(0,1,3)$ & $(0,2,6)$ & $(0,3,2)$ & $(0,4,5)$ & $(0,5,1)$ & $(0,6,4)$ \\
\hline$(1,0,2)$ & $(1,1,5)$ & $(1,2,1)$ & $(1,3,4)$ & $(1,4,0)$ & $(1,5,3)$ & $(0,6,6)$ \\
\hline$(2,0,4)$ & $(2,1,0)$ & $(2,2,3)$ & $(2,3,6)$ & $(2,4,2)$ & $(2,5,5)$ & $(2,6,1)$ \\
\hline$(3,0,6)$ & $(3,1,2)$ & $(3,2,5)$ & $(3,3,1)$ & $(3,4,4)$ & $(3,5,0)$ & $(3,6,3)$ \\
\hline$(4,0,1)$ & $(4,1,4)$ & $(4,2,0)$ & $(4,3,3)$ & $(4,4,6)$ & $(4,5,2)$ & $(4,6,5)$ \\
\hline$(5,0,3)$ & $(5,1,6)$ & $(5,2,2)$ & $(5,3,5)$ & $(5,4,1)$ & $(5,5,4)$ & $(5,6,0)$ \\
\hline$(6,0,5)$ & $(6,1,1)$ & $(6,2,4)$ & $(6,3,0)$ & $(6,4,3)$ & $(6,5,6)$ & $(6,6,2)$ \\
\hline
\end{tabular}


by the $2 \times 2 \times 2$ array consisting of the following two planes:

\begin{tabular}{|l|l|}
\hline 0 & 1 \\
\hline 2 & 3 \\
\hline
\end{tabular}$\quad$\begin{tabular}{|l|l|}
\hline 4 & 5 \\
\hline 6 & 7 \\
\hline
\end{tabular}

and furthermore replace each 1 by the $2 \times 2 \times 2$ array consisting of the planes:

\begin{tabular}{|c|c|}
\hline 8 & 9 \\
\hline 10 & 11 \\
\hline
\end{tabular}$\quad$\begin{tabular}{|c|c|}
\hline 12 & 13 \\
\hline 14 & 15 \\
\hline
\end{tabular}

The result is a 4 -interleaved array $A_{t}$. It is easy to see that the interleaving degree of $A_{t}$ is 16 , which is $2^{3}=8$ times the interleaving degree that we had for $A_{t / 2}$.

Assuming the availability of an optimal interleaving scheme, namely, a construction meeting the lower bound of Theorem 3.1 for a certain $t$, we can prove the following result.

Proposition 3.2: Given an optimal three-dimensional $t$ interleaved array $A_{t}$, application of Construction 3.2 produces a $2 t$-interleaved array $A_{2 t}$ with interleaving degree $2 t$ away from the lower bound when $t$ is even, and $6 t$ away from the lower bound when $t$ is odd.

Proof: We will prove the proposition for even $t$. The odd case can be proved similarly. By Theorem 3.1, the interleaving degree of the optimal $t$-interleaved array is

$$
\operatorname{deg} A_{t}=\frac{1}{6}\left(t^{3}+2 t\right) \text {. }
$$

Hence the degree of the $2 t$-interleaved array resulting from Construction 3.2 is given by:

$$
\operatorname{deg} A_{2 t}=2^{3} \operatorname{deg} A_{t}=\frac{8\left(t^{3}+2 t\right)}{6}=\frac{(2 t)^{3}+2(2 t)}{6}+2 t .
$$

We include the interleaving degrees resulting from Construction 3.2 in Table I. Notably, this construction improves upon Construction 3.1 for the case $t=12$.

In Construction 3.2, we substituted a $2 \times 2 \times 2$ array for each label in $A_{t}$ to obtain a $2 t$-interleaved array $A_{2 t}$. This idea can be easily generalized to the substitution of a $c \times c \times c$ array for each label in $A_{t}$. In fact, it is not difficult to see that this generalized version of Construction 3.2 is equivalent to scaling a $t$-interleaved array by an integer constant $c$ to obtain a $c t$-interleaved array. In three dimensions, the interleaving degree increases by a factor of $c^{3}$. This notion of scaling in the context of interleaving schemes will become apparent when we discuss lattice interleavers in the next section.

\section{The Sphere-Packing Bound is not Exact}

We have seen in Section II that the sphere-packing lower bound is exact in two dimensions: namely, for all $t$, this bound correctly predicts the minimum possible interleaving degree of a $t$-interleaved array. Herein, we show by a counterexample that the sphere-packing bound ceases to be exact in three dimensions. According to Table $\mathrm{I}$, the first value of $t$ for which this might happen is $t=5$, and we will prove that, indeed, a three-dimensional interleaving scheme with interleaving degree of $\frac{1}{6}\left(5^{3}+5 \cdot 5\right)=25$ does not exist.
This will follow from a more general result that an optimal $n$-dimensional $t$-interleaving scheme implies a close-packing of the $L_{1}$-spheres $\mathcal{S}_{n, t}$, along with a detailed analysis of a certain proof presented in [15]. But first, we need to introduce some notation.

Given a subset $\mathcal{S} \subseteq \mathbb{Z}^{n}$, we define the density of $\mathcal{S}$ as the ratio of the number of points in $\mathcal{S}$ to the number of points in $\mathbb{Z}^{n}$ contained in an open cube of side $M$, in the limit as $M \rightarrow \infty$ (provided the limit exists). Thus if $\Phi_{M}$ denotes the cube of side $M$ centered at the origin, the density $\Delta(\mathcal{S})$ of $\mathcal{S}$ is given by:

$$
\Delta(\mathcal{S}) \stackrel{\text { def }}{=} \lim _{M \rightarrow \infty} \frac{\left|\mathcal{S} \cap \Phi_{M}\right|}{M^{n}} .
$$

It is obvious that $\Delta(\mathcal{S})=0$ for any finite set $\mathcal{S}$, but we will be primarily interested in infinite sets in this subsection. In particular, we consider infinite arrangements of the $L_{1}$ spheres $\mathcal{S}_{n, t}$, defined in the previous section. Here, we will need a slightly more precise definition of these spheres. Given two points $x=\left(x_{1}, x_{2}, \cdots, x_{n}\right)$ and $y=\left(y_{1}, y_{2}, \cdots, y_{n}\right)$ in $\mathbb{R}^{n}$, we let

$$
d_{1}(x, y) \stackrel{\text { def }}{=}\left|x_{1}-y_{1}\right|+\left|x_{2}-y_{2}\right|+\cdots+\left|x_{n}-y_{n}\right|
$$

denote the $L_{1}$ distance between them. Then, for odd $t$, the $L_{1}$-sphere $\mathcal{S}_{n, t}$ centered at a point $x \in \mathbb{Z}^{n}$ may be defined as

$$
\mathcal{S}_{n, t}(x) \stackrel{\text { def }}{=}\left\{x^{\prime} \in \mathbb{Z}^{n}: 2 d_{1}\left(x, x^{\prime}\right) \leq t-1\right\} .
$$

Now let $\mathcal{S}$ be an infinite arrangement of disjoint spheres of this kind. We shall say that $\mathcal{S}$ is a tiling if every point of $\mathbb{Z}^{n}$ is contained in some sphere in $\mathcal{S}$. We say that $\mathcal{S}$ is a close-packing if $\Delta(\mathcal{S})=1$. Notice that a tiling is also a closepacking, but not necessarily vice versa, since the definition of density involves a limiting argument. There could be an infinite number of points not contained in a close-packing $\mathcal{S}$, as long as this number grows as $o\left(M^{n}\right)$.

For even $t$, it is more convenient to consider a coset of the integer lattice $\mathbb{Z}^{n}$, rather than the lattice $\mathbb{Z}^{n}$ itself. Specifically, let $\xi=\left(\frac{1}{2}, 0,0, \cdots, 0\right)$. Then, for even $t$, the sphere $\mathcal{S}_{n, t}$ centered at a point $x \in \mathbb{Z}^{n}$ may be defined as

$$
\mathcal{S}_{n, t}(x) \stackrel{\text { def }}{=}\left\{x^{\prime} \in \xi+\mathbb{Z}^{n}: 2 d_{1}\left(x, x^{\prime}\right) \leq t-1\right\} .
$$

Notice that the $L_{1}$-radius of $\mathcal{S}_{n, t}$ is always $\frac{1}{2}(t-1)$, regardless of whether $t$ is odd or even. Furthermore, replacing $\mathbb{Z}^{n}$ with $\xi+\mathbb{Z}^{n}$, the notation introduced in the foregoing paragraph, in particular the terms tiling and close-packing, extends in the obvious way.

We say that an $n$-dimensional $t$-interleaved array $A_{n, t}$ is optimal if it attains the sphere-packing bound with equality, namely, if $\operatorname{deg} A_{n, t}=\left|\mathcal{S}_{n, t}\right|$. We can always assume without loss of generality that the array $A_{n, t}$ is sufficiently large. Equivalently, we could simply consider an infinite array whose elements are indexed by the integer lattice $\mathbb{Z}^{n}$ if $t$ is odd, or by the coset $\xi+\mathbb{Z}^{n}$ if $t$ is even. We will refer to such infinite arrays as interleaving schemes.

Lemma 3.3: For all $n$ and $t$, the existence of an optimal interleaving scheme $A_{n, t}$ implies the existence of a closepacking of the $L_{1}$-spheres $\mathcal{S}_{n, t}$. 
Proof: Let $m$ denote the degree of $A_{n, t}$ so that $m=|\mathcal{S}|_{n, t}$ and $A_{n, t}$ is labeled by the integers $0,1, \cdots, m-1$. For each $a=0,1, \cdots, m-1$, let $\mathcal{S}_{a}$ denote the set of points in $A_{n, t}$ labeled by the integer $a$. If $x$ and $x^{\prime}$ are two points in $\mathcal{S}_{a}$, then obviously $d_{1}\left(x, x^{\prime}\right) \geq t$ since otherwise $A_{n, t}$ is not $t$-interleaved (as shown in Theorem 2.1.). Now let $\mathcal{S}_{a}^{*}$ be the infinite arrangement of the $L_{1}$-spheres $\mathcal{S}_{n, t}$, centered at the points of $\mathcal{S}_{a} \subset \mathbb{Z}^{n}$ for odd $t$, or at the points of $\xi+\mathcal{S}_{a} \subset \mathbb{Z}^{n}$ for even $t$. Since the $L_{1}$-radius of $\mathcal{S}_{n, t}$ is $\frac{1}{2}(t-1)$, the triangle inequality (in the $L_{1}$ metric) implies that all the spheres in $\mathcal{S}_{a}^{*}$ are disjoint. This further shows that for each point in $\mathcal{S}_{a}$, there are precisely $m=\left|\mathcal{S}_{n, t}\right|$ points in $\mathcal{S}_{a}^{*}$. Hence for all $a=0,1, \cdots, m-1$, we have

$$
\Delta\left(\mathcal{S}_{a}\right)=\frac{\Delta\left(\mathcal{S}_{a}^{*}\right)}{m} \leq \frac{1}{m} .
$$

On the other hand, the sets $\mathcal{S}_{0}, \mathcal{S}_{1}, \cdots, \mathcal{S}_{m-1}$ are disjoint, and taken together constitute a tiling of $\mathbb{Z}^{n}$, or of $\xi+\mathbb{Z}^{n}$ if $t$ is even. This implies

$$
\Delta\left(\mathcal{S}_{0}\right)+\Delta\left(\mathcal{S}_{1}\right)+\cdots+\Delta\left(\mathcal{S}_{m-1}\right)=1
$$

It now follows from (6) that (5) must hold with equality, namely, $\Delta\left(\mathcal{S}_{a}\right)=1 / m$ for all $a=0,1, \cdots, m-1$. But then the first equality in (5) further implies that $\Delta\left(\mathcal{S}_{a}^{*}\right)=1$. Thus each of the sets $\mathcal{S}_{0}^{*}, \mathcal{S}_{1}^{*}, \cdots, \mathcal{S}_{m-1}^{*}$ is a close-packing of the $L_{1}$-spheres $\mathcal{S}_{n, t}$.

We now analyze the proof of a result obtained by Golomb and Welch [15]. Golomb and Welch [15, Theorem 5] show that the sphere $\mathcal{S}_{3, \tilde{5}}$ does not tile the integer lattice $\mathbb{Z}^{3}$, but in our context we need a slightly stronger result that a closepacking of the spheres $\mathcal{S}_{3,5}$ does not exist. This result follows by looking at the proof in [15] closely, and observing that it employs only local arguments. Specifically, the proof of Theorem 5 in [15] actually establishes the following. Let $\mathcal{S}$ be an arrangement of disjoint spheres $\mathcal{S}_{3, \tilde{3}}$, such that one of them is centered at the origin $(0,0,0)$. Further, let $\Theta_{0}$ be the following set of eight points:

$$
\begin{aligned}
\Theta_{0}=\{(2,1,0),(2,-1,0),(1,1,1),(1,2,1),(1,1,-1), \\
\\
(1,2,-1),(2,2,-2)(2,3,-2)\}
\end{aligned}
$$

and let $\Gamma$ be the isometry group of order $2^{3} \cdot 3 !=48$, consisting of coordinate permutations and sign changes in $\mathbb{R}^{3}$. Let $\Theta_{0}, \Theta_{1}, \cdots, \Theta_{47}$ be the orbit of $\Theta_{0}$ under $\Gamma$. Then it is shown in [15] that at least one of the sets $\Theta_{0}, \Theta_{1}, \cdots, \Theta_{47}$ contains at least one point which is not in $\mathcal{S}$.

Notice that $\Theta_{0}, \Theta_{1}, \cdots, \Theta_{47}$ are all subsets of the open cube of side $M=7$ centered at the origin. Hence we can prove the nonexistence of a close-packing of the spheres $\mathcal{S}_{3,5}$ as follows. We partition $\mathbb{R}^{3}$ into disjoint open cubes of side $M=11$, with one of them centered at the origin. Obviously, each of these cubes contains exactly $11^{3}=1331$ points of $\mathbb{Z}^{3}$. Now let $\mathcal{S}$ be an arbitrary arrangement of disjoint spheres $\mathcal{S}_{3,5}$. It is easy to see that each of the cubes of side $M=11$ in our partition contains at most 1330 points of $\mathcal{S}$. Indeed, consider the point at the center of such a cube $\Phi$. If this point is not in $\mathcal{S}$, we are done. Otherwise, some $\mathcal{S}_{3,5}$ sphere in $\mathcal{S}$ contains this point. Let $x$ be the center of this sphere. We can now consider the cube $\Phi^{\prime}$ of side $M^{\prime}=7$, centered at $x$. It is obvious that $\Phi^{\prime} \subset \Phi$, and according to the aforementioned proof in Golomb and Welch [15] at least one point in $\Phi^{\prime}$ is not contained in $\mathcal{S}$. It follows that $\Delta(\mathcal{S}) \leq 1330 / 1331<1$. Thus we have established

Theorem 3.4: The sphere-packing lower bound is not exact for $n=3$ and $t=5$.

We observe that the techniques described in this subsection could be, in principle, used to show that the sphere-packing bound is unattainable for other values of $n$ and $t$. In fact, [15, Appendix] may be used as an argument alternative to our analysis of [15, Theorem 5], although such an argument would be much less concrete. In particular, [15, Appendix] shows that if a close-packing of certain shapes exists then these shapes must admit a tiling. We also observe that Lemma 3.3 in conjunction with [15, proof of Theorem 6] imply that, in three dimensions, the sphere-packing bound is unattainable for all sufficiently large $t$. In fact, it is possible to show that this statement is true for any fixed dimension $n>2$.

\section{LATTICE INTERLEAVERS}

In this section we introduce multidimensional interleaving schemes based on lattices. We will refer to such schemes as lattice interleavers. We observe that Constructions 2.1, 2.2, 3.1 , and 3.2-that is, all the constructions presented in the foregoing two sections-are actually special cases of lattice interleavers. This will enable us, in particular, to provide a concise proof for Theorems 2.2 and 2.3. Furthermore, we will show that for three-dimensional lattice interleavers, there is a much stronger lower bound on the interleaving degree than Theorem 3.1. This bound is based on the fundamental result of Minkowski [23], [24] on lattice packings of octahedra in the three-dimensional Euclidean space $\mathbb{R}^{3}$. Minkowski [16], [24] also constructs a lattice which attains the lower bound with equality. Whenever $t \equiv 0 \bmod 6$, this lattice directly produces the optimal lattice interleaver. For $t \neq \equiv \bmod 6$, we construct lattice interleavers using certain approximations of the Minkowski lattice. This reproduces all of the results listed in Table I, and improves upon this table in two cases.

\section{A. Preliminaries}

We start with some notation and definitions. Henceforth, we will say that an interleaving scheme which guarantees that every cluster of size $\leq t$ is labeled distinctly is an interleaver of strength $t$. Suppose that we can partition $\mathbb{Z}^{n}$ into $m$ cosets $\mathcal{Z}_{0}, \mathcal{Z}_{1}, \cdots, \mathcal{Z}_{m-1}$, such that the minimum $L_{1}$-distance within each coset is at least $t$. Then, by assigning a different integer to each coset, such partition of $\mathbb{Z}^{n}$ produces an interleaving scheme of degree $m$ and strength $t$. The easiest way to partition $\mathbb{Z}^{n}$ into cosets, such that all of them have the same minimum distance, is to consider the translates of a sublattice $\Lambda$ of $\mathbb{Z}^{n}$.

A lattice $\Lambda$ is a discrete, nowhere dense, additive subgroup of the real $n$-space $\mathbb{R}^{n}$. Without loss of generality, we can 
assume that

$$
\Lambda=\left\{u_{1} v_{1}+u_{2} v_{2}+\cdots+u_{n} v_{n}: u_{1}, u_{2}, \cdots, u_{n} \in \mathbb{Z}\right\}
$$

where $\left\{v_{1}, v_{2}, \cdots, v_{n}\right\}$ is a set of linearly independent vectors in $\mathbb{R}^{n}$. A lattice $\Lambda$ defined by (7) is a sublattice of $\mathbb{Z}^{n}$ if and only if $\left\{v_{1}, v_{2}, \cdots, v_{n}\right\} \subset \mathbb{Z}^{n}$, and we will be interested in sublattices of $\mathbb{Z}^{n}$ unless stated otherwise. The vectors $v_{1}, v_{2}, \cdots, v_{n}$ are called a basis for $\Lambda$, and the $n \times n$ matrix

$$
\boldsymbol{G}=\left[\begin{array}{cccc}
v_{11} & v_{12} & \cdots & v_{1 n} \\
v_{21} & v_{22} & \cdots & v_{2 n} \\
\vdots & \vdots & \cdots & \vdots \\
v_{n 1} & v_{n 2} & \cdots & v_{n n}
\end{array}\right]
$$

having these vectors as its rows is said to be a generator matrix for $\Lambda$. The volume of a lattice $\Lambda$, denoted $V(\Lambda)$, is inversely proportional to the number of lattice points per unit volume. More precisely, the quantity $V(\Lambda)$ may be defined as the volume of the fundamental parallelotope $\Pi(\Lambda)$, which is given by

$$
\begin{array}{r}
\Pi(\Lambda) \stackrel{\text { def }}{=}\left\{\theta_{1} v_{1}+\theta_{2} v_{2}+\cdots+\theta_{n} v_{n}: 0 \leq \theta_{i}<1\right. \\
\text { for } i=1,2, \cdots, n\} .
\end{array}
$$

The significance of volume in our context derives from the following well-known [9] observation: the index of a sublattice $\Lambda$ of $\mathbb{Z}^{n}$ in $\mathbb{Z}^{n}$ is equal to its volume, that is, the order of the group $\mathbb{Z}^{n} / \Lambda$ is precisely $V(\Lambda)$. There is a simple expression for the volume of $\Lambda$, namely:

$$
V(\Lambda)=|\operatorname{det} \boldsymbol{G}| \text {. }
$$

Thus given a lattice $\Lambda \subseteq \mathbb{Z}^{n}$ of volume $V(\Lambda)=m$, the integer lattice $\mathbb{Z}^{n}$ can be partitioned into $m$ additive cosets $\Lambda, x_{1}+\Lambda, \cdots, x_{m-1}+\Lambda$ for some $x_{1}, x_{2}, \cdots, x_{m-1} \in \mathbb{Z}^{n}$. The coset representatives in this partition can be easily found as follows:

$$
\left\{\mathbf{0}, x_{1}, x_{2}, \cdots, x_{m-1}\right\}=\mathbb{Z}^{n} \cap \Pi(\Lambda) .
$$

This is certainly not the only possible choice of coset representatives, but it will suffice for our purposes. In two and three dimensions, there are efficient algorithms [27] for computing the coset representatives, given a generator matrix $\boldsymbol{G}$ for $\Lambda$, using the expression in (8).

Since a lattice $\Lambda$ is an additive group, the minimum $L_{1}$ distance between points of $\Lambda$ is equal to the minimum $L_{1}$-norm of a nonzero point in $\Lambda$. In what follows, we let

$$
\mu_{1}(\Lambda) \stackrel{\text { def }}{=} \min _{\substack{x \in \Lambda \\ x \neq \mathbf{0}}} d_{1}(x, \mathbf{0})
$$

denote the minimum $L_{1}$-norm of $\Lambda$. According to the foregoing discussion, $\mu_{1}(\Lambda)$ is the strength of interleaving in the $n$-dimensional interleaving scheme based on $\mathbb{Z}^{n} / \Lambda$.

This is essentially all one needs to know about lattices in the context of this paper. We refer the interested reader to Conway and Sloane [9] for an encyclopedic treatment of this subject. Herein, we can summarize all of the above with the following theorem.
Theorem 4.1: Let $\Lambda$ be a sublattice of $\mathbb{Z}^{n}$ generated by an $n \times n$ integer matrix $\boldsymbol{G}$. Then a partition of $\mathbb{Z}^{n}$ into cosets of $\Lambda$ produces an $n$-dimensional interleaving scheme of strength $t=\mu_{1}(\Lambda)$ and degree $m=V(\Lambda)=|\operatorname{det} G|$.

We observe that in two and three dimensions, the minimum $L_{1}$-norm $t$ of an arbitrary sublattice $\Lambda$ of $\mathbb{Z}^{n}$ can be computed in time $O\left(t^{2}\right)$ or $O\left(t^{3}\right)$, respectively. In three dimensions, this can be done by simply going through all the nonzero points $(x, y, z)$ with integer coordinates that are contained in the octahedron:

$$
|x|+|y|+|z| \leq t-1
$$

and checking whether any one of them is contained in $\Lambda$. Since negation preserves the $L_{1}$-norm and constitutes a symmetry of every lattice $\Lambda$, we may in fact consider only half the octahedron. Namely, we can assume that $x \geq 0$ in (10), and if $x=0$ then $y \geq 0$, and finally if $x=y=0$ then $z>0$. Thus verifying that $\mu_{1}(\Lambda) \geq t$ for an arbitrary sublattice of $\mathbb{Z}^{3}$ involves considering at most $\frac{1}{3}(t-1)\left(2 t^{2}-t+3\right)$ points. It follows that for any given sublattice $\Lambda$ of $\mathbb{Z}^{2}$ or $\mathbb{Z}^{3}$, both the degree and the strength of the corresponding interleaving scheme can be efficiently computed.

\section{B. Lattice Interleavers in Two Dimensions}

In this subsection, we describe certain specific two-dimensional lattice interleavers, in particular those that correspond to the constructions presented in Section II. First, consider the lattices $\Lambda_{e, t}$ and $\Lambda_{O, t}$ generated by:

$$
\boldsymbol{G}_{e, t}=\left[\begin{array}{rr}
k & -k \\
k & k
\end{array}\right] \quad \text { and } \quad \boldsymbol{G}_{o, t}=\left[\begin{array}{cc}
k+1 & -k \\
k & k+1
\end{array}\right]
$$

for even $t=2 k$ and odd $t=2 k+1$, respectively. These lattices are illustrated in Fig. 1 for $t=6$ and $t=7$.

Theorem 4.2: Lattice interleavers based on the lattices $\Lambda_{e, t}$ and $\Lambda_{o, t}$ are optimal.

Proof: According to Theorem 4.1, the interleaving degree is given by the volume of $\Lambda_{e, t}$ and $\Lambda_{o, t}$. Trivially, we have:

$$
\begin{aligned}
& V\left(\Lambda_{e, t}\right)=\operatorname{det} \boldsymbol{G}_{e, t}=2 k^{2}=\frac{1}{2} t^{2} \\
& V\left(\Lambda_{o, t}\right)=\operatorname{det} \boldsymbol{G}_{o, t}=(k+1)^{2}+k^{2}=\frac{1}{2}\left(t^{2}+1\right) .
\end{aligned}
$$

Hence, according to Theorems 2.1 and 4.1, all we need to show is that the minimum $L_{1}$-norm of both lattices is at least $t$. For even $t=2 k$, this follows directly by observing that $\Lambda_{e, t}$ is just a scaling by a factor of $k$ of the familiar checkerboard lattice $D_{2}$ generated by $(1,1)$ and $(1,-1)$, whose minimum $L_{1}$-norm is obviously 2 . For odd $t=2 k+1$, we write a generic nonzero point $(x, y) \in \Lambda_{o, t}$ as follows:

$$
\begin{aligned}
& x=a(k+1)+b k=k(a+b)+a \\
& y=-a k+b(k+1)=k(b-a)+b
\end{aligned}
$$

for some integers $a$ and $b$, not both zero. Since negation is a symmetry of $\Lambda_{o, t}$, we may assume without loss of generality (w.l.o.g.) that $a \geq 0$. If $a \geq 1$ and $b \geq 1$, then $x \geq 2 k+1=t$. If $a \geq 1$ and $\bar{b} \leq-1$, then $-y \geq 2 k+\overline{1}=t$. This leaves the case $a=0$, in which case we can further assume 


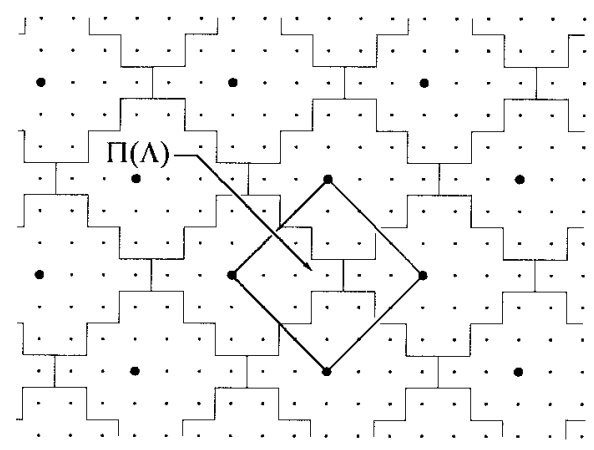

(a)

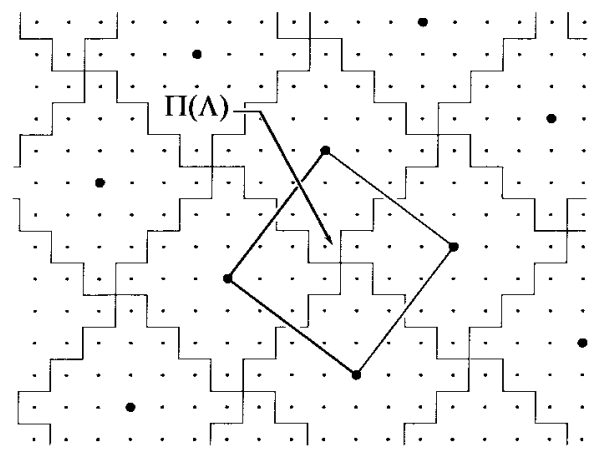

(b)

Fig. 1. Optimal lattice interleavers in two dimensions. (a) Even $t=6$. (b) Odd $t=7$.

w.l.o.g. that $b \geq 1$. Thus $x=b k$ and $y=b(k+1)$, so that $|x|+|y|=b(2 \bar{k}+1) \geq t$.

It is easy to see (cf. Example 2.4) that Construction 2.2 is precisely the lattice interleaver based on $\Lambda_{e, t}$. It is also easy to see that Theorems 2.2 and 2.3 produce lattice interleavers based on the lattices $\Lambda_{O, t}^{*}$ and $\Lambda_{e, t}^{*}$, generated by

$$
\begin{aligned}
& \boldsymbol{G}_{o, t}^{*}=\left[\begin{array}{cc}
1 & b \\
0 & m
\end{array}\right]=\left[\begin{array}{cc}
1 & t \\
0 & \frac{1}{2}\left(t^{2}+1\right)
\end{array}\right]=\left[\begin{array}{cc}
1 & 2 k+1 \\
0 & (k+1)^{2}+k^{2}
\end{array}\right] \\
& \boldsymbol{G}_{e, t}^{*}=\left[\begin{array}{cc}
1 & b \\
0 & m
\end{array}\right]=\left[\begin{array}{cc}
1 & t+1 \\
0 & \frac{1}{2} t^{2}
\end{array}\right]=\left[\begin{array}{cc}
1 & 2 k+1 \\
0 & 2 k^{2}
\end{array}\right]
\end{aligned}
$$

for odd $t=2 k+1$ and even $t=2 k$, respectively. It is not difficult to show that $\Lambda_{o, t}^{*}$ and $\Lambda_{e, t}^{*}$ are just variations of $\Lambda_{o, t}$ and $\Lambda_{e, t}$, respectively. We illustrate this for the case of odd $t$. The operation of negating the first coordinate is an isometry in $\mathbb{R}^{2}$ with respect to the $L_{1}$ metric: it is clear that this operation does not change the volume or the minimum $L_{1}$ distance of a lattice. Negating the first coordinate of $\Lambda_{0, t}$, we obtain a lattice generated by

$$
\left[\begin{array}{l}
v_{1} \\
v_{2}
\end{array}\right]=\left[\begin{array}{cc}
-(k+1) & -k \\
-k & k+1
\end{array}\right]
$$

Now, we observe that

$$
v_{2}-v_{1}=(1,2 k+1)
$$

and

$$
(k+1) v_{2}-k v_{1}=\left(0,(k+1)^{2}+k^{2}\right)
$$

so this lattice is precisely $\Lambda_{o, t}^{*}$. A similar, although more complicated argument, shows that $\Lambda_{e, t}^{*}$ is essentially a variation of $\Lambda_{e, t}$ with the same volume and minimum $L_{1}$-distance. Therefore, the proof of Theorem 4.2 also establishes Theorems 2.2 and 2.3 .

\section{Lattice Interleavers in Three Dimensions}

In three dimensions, we observe that Construction 3.1 also produces lattice interleavers. These interleavers are based on lattices generated by a matrix of the following special form:

$$
G=\left[\begin{array}{ccc}
1 & 0 & b \\
0 & 1 & c \\
0 & 0 & m
\end{array}\right] .
$$

It is obvious that $\operatorname{det} G=m$, and hence the interleaving degree is precisely $m$. The special form of $G$ makes it possible to compute the minimum $L_{1}$ distance of the lattice generated by $\boldsymbol{G}$ in a manner different from (10), as explained in Section III.

Finally, Construction 3.2 is equivalent to scaling a lattice interleaver (or any partition of $\mathbb{Z}^{3}$ into cosets) by a factor of 2 . Obviously, this increases the volume by a factor of $2^{3}$ while increasing the interleaving degree by a factor of 2 . This can be generalized to scaling by any constant $c$. We shall see in a later subsection that such scaling operation produces optimal lattice interleavers whenever $t \equiv 0 \bmod 6$.

We now describe a lower bound on the interleaving degree of lattice interleavers in three dimensions, that is generally much stronger than the sphere-packing lower bound. The bound is based on the following fundamental result of Minkowski [24], dating back to 1904.

Theorem 4.3 (Minkwoski 1904): Let $\Xi_{t} \subset \mathbb{R}^{3}$ be the regular three-dimensional octahedron of $L_{1}$-radius $t$, defined by

$$
\Xi_{t} \stackrel{\text { def }}{=}\left\{(x, y, z) \in \mathbb{R}^{3}:|x|+|y|+|z|<t\right\} .
$$

Then the density of any lattice packing of disjoint translates of $\Xi_{t}$ is at most $18 / 19$.

It is easy to see by straightforward integration that the volume of the octahedron $\Xi_{t}$ is $\frac{4}{3} t^{3}$. This immediately establishes the following lower bound on the interleaving degree of three-dimensional lattice interleavers.

Theorem 4.4: Let $A$ be a three-dimensional interleaving scheme of strength $t$ based on a lattice $\Lambda \subseteq \mathbb{Z}^{3}$. Then

$$
\operatorname{deg} A \geq\left\lceil\frac{19}{108} t^{3}\right\rceil
$$

Proof: Let $\Lambda$ be a sublattice of $\mathbb{Z}^{3}$ with $\mu_{1}(\Lambda) \geq t$. Then octahedra of $L_{1}$-radius $t / 2$ centered around the points of $\Lambda$ are disjoint. The volume of such an octahedron $\Xi_{t / 2}$ is

$$
V\left(\Xi_{t / 2}\right)=\frac{4}{3}(t / 2)^{3}=\frac{1}{6} t^{3} .
$$

Since the number of lattice points per unit volume is $1 / V(\Lambda)$, the density of the resulting lattice packing of octahedra is $V\left(\Xi_{t / 2}\right) / V(\Lambda)$. This density cannot exceed the Minkowski bound in Theorem 4.3. Hence we have

$$
\frac{V\left(\Xi_{t / 2}\right)}{V(\Lambda)}=\frac{\frac{1}{6} t^{3}}{V(\Lambda)} \leq \frac{18}{19}
$$


By the discussion in Section IV-A, the interleaving degree of the three-dimensional interleaving scheme $A$ based on $\Lambda$ is given by $\operatorname{deg} A=V(\Lambda)$, and the theorem follows directly from (11).

The lower bound of Theorem 4.4 is stronger than the spherepacking lower bound of Theorem 3.1 for all $t \geq 8$. The values of this bound for $t=2,3, \cdots, 18$ are listed in Table II.

A well-known conjecture [29] says that the Minkowski bound of $18 / 19$ on the density of lattice packings of octahedra in fact holds for any octahedral packing in $\mathbb{R}^{3}$. If this conjecture is true, then it is easy to see that the lower bound of Theorem 4.4 applies to any three-dimensional interleaving scheme of strength $t$.

\section{Interleavers Based on the Minkowski Lattice}

In his seminal papers [23], [24], Minkowski not only established a lower bound on the density of lattice packings of octahedra, but also exhibited the lattice which attains this bound with equality. Specifically, Minkowski [24] studied the integer span of of the following matrix:

$$
\mathfrak{G}=\left[\begin{array}{rrr}
-\frac{1}{3} & \frac{1}{2} & \frac{1}{6} \\
\frac{1}{6} & -\frac{1}{3} & \frac{1}{2} \\
\frac{1}{2} & \frac{1}{6} & -\frac{1}{3}
\end{array}\right] .
$$

We shall refer to the lattice generated by the matrix $\mathfrak{G}$ in (12) as the Minkowski lattice, and denote it by $\mathfrak{M}$. It is easy to see that $V(\mathfrak{M})=\operatorname{det} \mathfrak{G}=19 / 108$, and Minkowski [24] showed that $\mu_{1}(\mathfrak{M})=1$, which can be also verified directly. Hence $\mathfrak{M}$ produces a lattice packing of octahedra $\Xi_{1 / 2}$ of optimal density $V\left(\Xi_{1 / 2}\right) / V(\mathfrak{M})=18 / 19$.

Here, we are interested in packings of octahedra of $L_{1}$ radius $t / 2$, for integer $t$. Such packings can be obtained by scaling the Minkowski lattice by a factor of $t$. It is obvious from (12) that scaling $\mathfrak{M}$ by a factor of $t$ produces a sublattice of $\mathbb{Z}^{3}$ if and only if $t \equiv 0 \bmod 6$. Thus we have

Theorem 4.5: For $t \equiv 0 \bmod 6$, scaling the Minkowski lattice by a factor of $t$ produces a three-dimensional lattice interleaver $A$ of strength $t$ and

$$
\operatorname{deg} A=\frac{19}{108} t^{3}
$$

This interleaver has the lowest possible degree among all lattice interleavers of strength $t$.

For $t \not \equiv 0 \bmod 6$, the lattice $\mathfrak{M}_{t}$ that results from scaling the Minkowski lattice by a factor of $t$ is still the optimal lattice packing of octahedra $\Xi_{t / 2}$, but it is not a sublattice of $\mathbb{Z}^{3}$. Hence $\mathfrak{M}_{t}$ cannot be used directly to produce an interleaving scheme of strength $t$.

A reasonable thing to do in this case is to approximate the scaled version of the Minkowski lattice by a sublattice of $\mathbb{Z}^{3}$. For example, for $t=11$, the lattice $\mathfrak{M}_{11}$ is generated by

$$
\mathfrak{G}_{11}=\left[\begin{array}{rrr}
-\frac{11}{3} & \frac{11}{2} & \frac{11}{6} \\
\frac{11}{6} & -\frac{11}{3} & \frac{11}{2} \\
\frac{11}{2} & \frac{11}{6} & -\frac{11}{3}
\end{array}\right] .
$$

Each of the three generators in $\mathfrak{G}_{11}$ is contained in a unit cube whose vertices are points of $\mathbb{Z}^{3}$, as depicted in Fig. 2. For instance, the first generator $v_{1}=\left(-\frac{11}{3}, \frac{11}{2}, \frac{11}{6}\right)$ is contained in the unit cube with vertices at

$$
\begin{aligned}
& (-4,5,1),(-4,5,2),(-4,6,1),(-4,6,2), \\
& (-3,5,1),(-3,5,2),(-3,6,1),(-3,6,2) .
\end{aligned}
$$

Each of the vertices of this cube may be used to approximate $v_{1}$; that is, rounding $v_{1}$ to any one of the eight points in (13) alters the geometry of $\mathfrak{M}_{11}$ only slightly. A similar approximation technique can be used for the remaining two generators in $\mathfrak{G}_{11}$.

In general, there are $2^{3}$ different ways to approximate each generator in $\mathfrak{G}_{t}$, and $8^{3}=512$ different ways to approximate the entire lattice $\mathfrak{M}_{t}$ in this manner. For each of the resulting 512 sublattices of $\mathbb{Z}^{3}$, we can verify in time $O\left(t^{3}\right)$ whether the interleaving strength is still $\geq t$, using the procedure described in Section IV-A. The interleaving degree can be straightforwardly computed as the determinant of the corresponding generator matrix. Thus we can find in time $O\left(t^{3}\right)$ the approximation of $\mathfrak{M}_{t}$ which has the lowest interleaving degree among all the approximations of interleaving strength at least $t$.

The results of this computation are compiled in Table II. We note that in many cases, more than one approximation of $\mathfrak{M}_{t}$ of strength $t$ attains the interleaving degree reported in Table II. However, we include only one set of generators in the table, which is selected arbitrarily if the best approximation is not unique. We observe that Table II improves upon the upper bounds of Table I in two cases, namely, for $t=9$ and $t=10$.

The results of Table II at least equal those of Table I in all cases but one, namely, for $t=13$. For $t=13$, the lowest interleaving degree obtained with first-order approximations of the Minkowski lattice is 422 . However, this can be reduced to the interleaving degree of 421 (same as in Table I), by considering second-order approximations. Namely, we can consider approximations of the three generators $v_{1}, v_{2}, v_{3}$ in $\mathfrak{G}_{t}$ by the $4^{3}=64$ points of $\mathbb{Z}^{3}$ contained in the cubes of side 3 centered at $v_{1}, v_{2}, v_{3}$. The corresponding set of generators for $t=13$ is

$$
(-4,7,2),(1,-5,7),(7,2,-4) \text {. }
$$

In principle, this approach could be extended by considering approximations of third-order, fourth-order, and so on. However, we quickly reach a point of diminishing returns. The case of $t=13$ above is the only one where we were able to find lattice interleavers better than first-order approximations of the Minkowski lattice. On the other hand, the complexity of computation increases rapidly. Already for the secondorder approximation, we have to consider $64^{3}=2^{18}$ different lattices.

We conclude this section by observing that the Minkowski lattice also provides the solution to the asymptotic problem of finding the best three-dimensional lattice interleavers of strength $\geq t$ for $t \rightarrow \infty$. Asymptotically, the lowest possible interleaving degree for lattice interleavers in three dimensions grows as $\frac{19}{108} t^{3}$. The lower bound is that of Theorem 4.2. As 


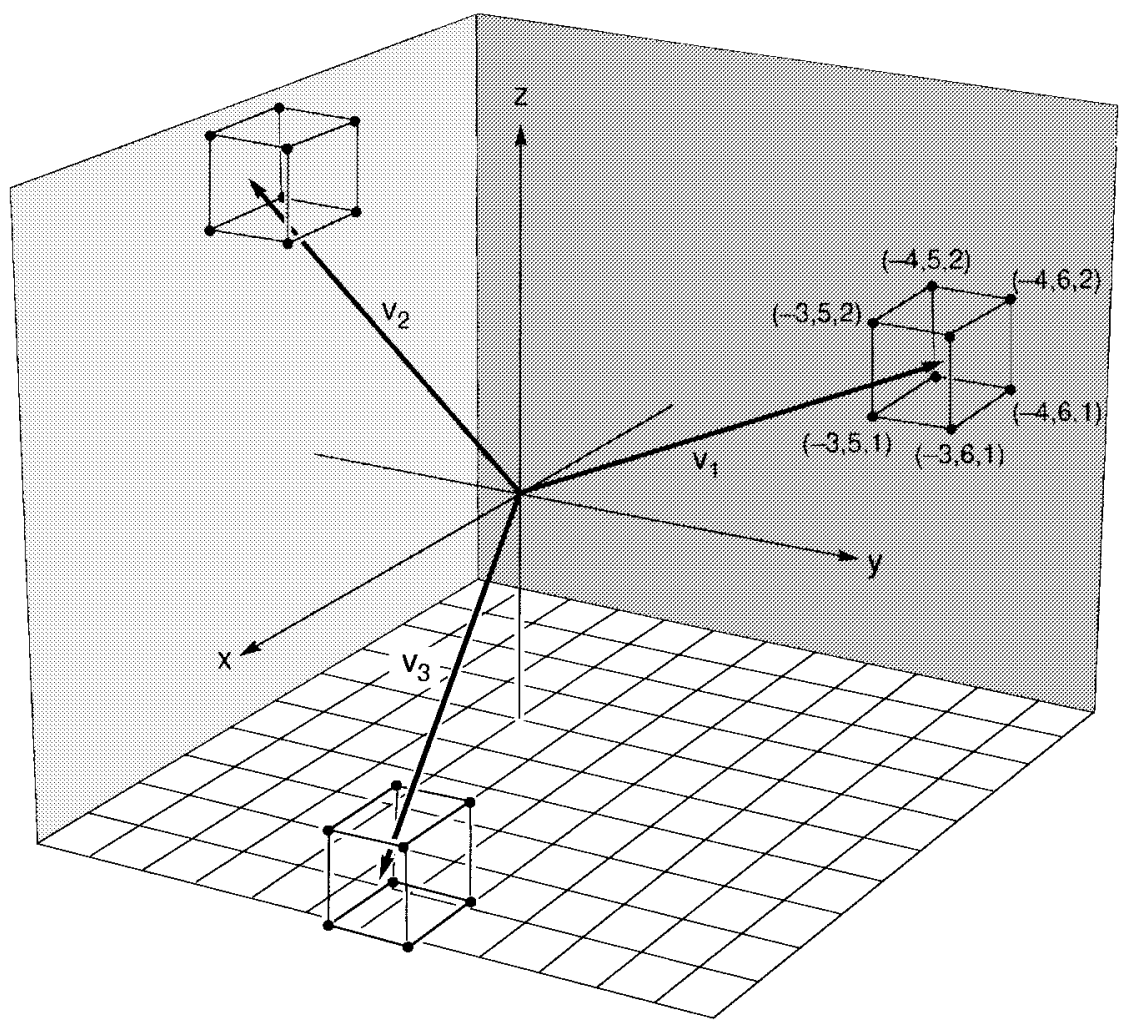

Fig. 2. Approximating the Minkowski lattice.

TABLE II

Bounds on the Interleaving Degree of Three-Dimensional Lattice InTERLEavers

\begin{tabular}{|c|c|c|c|c|}
\hline \multirow{2}{*}{$t$} & \multicolumn{2}{|c|}{ Lower Bounds } & \multicolumn{2}{|r|}{ Upper Bounds } \\
\hline & $\begin{array}{l}\text { Sphere } \\
\text { packing }\end{array}$ & Minkowski & $\begin{array}{c}\text { Minkowski } \\
\text { Lattice }\end{array}$ & generators \\
\hline 2 & 2 & 2 & 2 & $(-1,2,1),(1,-1,0),(0,1,-1)$ \\
\hline 3 & 7 & $\tilde{j}$ & 7 & $(-1,2,1),(1,-1,1),(1,1,-2)$ \\
\hline 4 & 12 & 12 & 12 & $(-1,2,1),(1,-1,2),(2,0,-2)$ \\
\hline 5 & 25 & 22 & 27 & $(-2,2,1),(1,-2,3),(3,1,-2)$ \\
\hline 6 & 38 & 38 & 38 & $(-2,3,1),(1,-2,3),(3,1,-2)$ \\
\hline 7 & 63 & 61 & 70 & $(-2,4,2),(1,-3,4),(3,1,-3)$ \\
\hline 8 & 88 & 91 & 92 & $(-2,4,2),(1,-3,4),(4,1,-3)$ \\
\hline 9 & 129 & 129 & 144 & $(-3,5,1),(1,-3,5),(5,1,-3)$ \\
\hline 10 & 170 & 176 & 178 & $(-3,5,2),(2,-3,5),(5,1,-4)$ \\
\hline 11 & 231 & 235 & 260 & $(-4,5,2),(2,-4,5),(6,2,-4)$ \\
\hline 12 & 292 & 304 & 304 & $(-4,6,2),(2,-4,6),(6,2,-4)$ \\
\hline 13 & 377 & 387 & 422 & $(-4,7,3),(2,-5,7),(6,2,-5)$ \\
\hline 14 & 462 & $48: 3$ & 486 & $(-1,7,3),(2,-5,7),(7,2,-5)$ \\
\hline 15 & 575 & 59.4 & 635 & $(-5,8,2),(2,-5,8),(8,2,-5)$ \\
\hline 16 & 688 & 721 & 724 & $(-5,8,3),(3,-5,8),(8,2,-6)$ \\
\hline 17 & 833 & 865 & 921 & $(-5,9,3),(2,-5,9),(9,2,-6)$ \\
\hline 18 & 978 & 1026 & 1026 & $(-6,9,3),(3,-6,9),(9,3,-6)$ \\
\hline
\end{tabular}


an upper bound, we can simply scale the Minkowski lattice $\mathfrak{M}$ by the smallest integer $\tau$, such that $\tau \geq t$ and $\tau \equiv 0 \bmod 6$. Since $\tau \leq t+5$, the resulting interleaving degree is

$$
\frac{19}{108} \tau^{3} \leq \frac{19}{108}\left(t^{3}+15 t^{2}+75 t+125\right)=\frac{19}{108} t^{3}+O\left(t^{2}\right) .
$$

Thus it is fair to say that, asymptotically, the problem of finding the best lattice interleaver in three dimensions is closed.

\section{ACKNOWLEDGMENT}

A. Vardy would like to acknowledge discussions with H. Edelsbrunner and D. B. West. He is also grateful to Hagit Itzkowitz for her invaluable help.

\section{REFERENCES}

[1] K. A. S. Abdel-Ghaffar, R. J. McEliece, and H. C. A. van Tilborg, "Twodimensional burst identification codes and their use in burst correction," IEEE Trans. Inform. Theory, vol. 34, pp. 494-504, 1988.

[2] C. de Almeida and R. Palazzo, "Two-dimensional interleaving using the Set partioning technique," in Proc. IEEE Int. Symp. Information Theory (Trondheim, Norway, 1994), p. 505.

[3] J. J. Ashley, M. Blaum, and B. H. Marcus, "Report on coding techniques for holographic storage,” IBM Res. Rep. RJ 10013, Mar. 1996.

[4] M. Blaum and J. Bruck, "Correcting of two-dimensional clusters by interleaving of symbols," in Proc. IEEE Int. Symp. Information Theory (Trondheim, Norway, 1994), p. 504.

[5] M. Blaum and P. G. Farrell, "Array codes for cluster-error correction," Electron. Lett., vol. 30, pp. 1752-1753, 1994.

[6] D. Brady and D. Psaltis, "Control of volume holograms," J. Opt. Soc. Amer., vol. 9A, pp. 1167-1177, 1992.

[7] W. J. Burke and P. Sheng, "Crosstalk noise from multiple thick-phase holograms," J. Appl. Phys., vol. 48, pp. 681-690, 1977.

[8] B. Chor, C. E. Leiserson, R. L. Rivest, and J. B. Shearer, "An application of number theory to the organization of raster-graphics memory," $J$. Assoc. Comput. Mach., vol. 33, pp. 86-104, 1986.

[9] J. H. Conway and N. J. A. Sloane, Sphere Packings, Lattices and Groups. New York: Springer-Verlag, 1988.

[10] P. Delsarte, "Bilinear forms over a finite field, with applications to coding theory," J. Combin. Theory, vol. 25-A, pp. 226-241, 1978.

[11] P. G. Farrell, "Array codes for correcting cluster-error patterns," in Proc. IEE Conf. Elect. Signal Processing (York, England, July 1982).

[12] E. M. Gabidulin, "Combinatorial metrics in coding theory," in Proc. IEEE Int. Symp. Information Theory (Budapest, Hungary, 1971), pp. 169-176.
[13] _ "Theory of codes with maximum rank distance," Probl. Pered. Inform., vol. 21, pp. 3-16, 1985 (in Russian).

[14] E. M. Gabidulin and V. V. Zanin, "Codes correcting array bursts," in Proc. Workshop Information Protection (Moscow, USSR, 1993), pp. 24-25.

[15] S. W. Golomb and L. R. Welch, "Perfect codes in the Lee metric and the packing of polyominoes," SIAM J. Appl. Math., vol. 18, pp. 302-317, 1970.

[16] H. Hancock, Minkowski Geometry of Numbers. New York: Macmillan, 1939.

[17] C. Gu, J. Hong, I. McMichael, R. Saxena, and F. Mok, "Cross-talklimited storange capacity of volume holographic memory," J. Opt. Soc. Amer., vol. 9A, pp. 1978-1987, 1992.

[18] J. F. Heanie, M. C. Bashaw, and L. Hesselink, "Volume holographic storage and retrieval of digital data," Sci. Mag., vol. 265, pp. 749-752, 1994.

[19] H. Imai, "Two-dimensional Fire codes," IEEE Trans. Inform. Theory, vol. IT-19, pp. 796-806, 1973.

[20] , "A theory of two-dimensional cyclic codes," Inform. Contr., vol. 34, pp. 1-21, 1977

[21] S. A. Lis and P. D. Henshaw, "Ultra-dense optical mass storage," Report prepared for USAF, AFSC, AD-A232 767, 1991.

[22] E. S. Maniloff and K. M. Johnson, "Maximized photorefractive holographic storage," J. Appl. Phys., vol. 70, pp. 4702-4715, 1991.

[23] H. Minkowski, "Quelques nouveaux théorémes sur l'approximation des quantités a l'aide de nombres rationnels," Bull. Sci. Mathematique, vol. 25, pp. 72-76, 1901

[24] — "Dichteste gitterförmige Lagerung kongruenter Körper," Nachrichten Ges. Wiss. Göttingen, pp. 311-355, 1904.

[25] F. H. Mok, "Angle-multiplexed storage of 5000 holograms in lithium niobate," Opt. Lett., vol. 18, p. 915, 1993.

[26] M. A. Neifeld and M. McDonald, "Error correction for increasing the usable capacity of photorefractive memories," Opt. Lett., vol. 19, p. 1483, 1994.

[27] F. P. Preparata and M. I. Shamos, Computational Geometry. New York: Springer-Verlag, 1985.

[28] T. R. N. Rao and E. Fujiwara, Error-Control Coding for Computer Systems. Englewood Cliffs, NJ: Prentice-Hall, 1989.

[29] C. A. Rogers, Packing and Covering. Cambridge, U.K.: Cambridge Univ. Press, 1964

[30] R. M. Roth, "Maximum-rank array codes and their application to crisscross error correction," IEEE Trans. Inform. Theory, vol. 37, pp. 328-336, 1991.

[31] G. T. Sincerbox, "Holographic storage re-visited," in Current Trends in Optics, C. Dainty, Ed. San Diego, CA: Academic, 1994.

[32] A. Vardy, M. Blaum, P. H. Siegel, and G. T. Sincerbox, "Conservative arrays: Multi-dimensional modulation codes for holographic recording," IEEE Trans. Inform. Theory, vol. 42, pp. 227-230, 1996.

[33] V. G. Vizing, "On an estimate of the chromatic class of a $p$-graph," Diskret. Analiz., vol. 3, pp. 25-30, 1964.

[34] D. B. West, Introduction to Graph Theory. Englewood Cliffs, NJ: Prentice-Hall, 1996. 